\title{
Fruit Image Segmentation Based on a Colour Perception Neural Network Inspired by the Retina Structure
}

\author{
Q.Y Sun \\ Key Laboratory of Opto Elecronic Science and Technology \\ for Medicine of Ministry of Education \\ College of Photonic and Electronic Engineering Fujian \\ Normal University \\ Fujian, China \\ Fujian Agriculture and Forestry University \\ Fujian, China \\ Q.X Wu \\ Key Laboratory of Opto Elecronic Science and Technology \\ for Medicine of Ministry of Education \\ College of Photonic and Electronic Engineering Fujian \\ Normal University \\ Fujian, China
}

\author{
X. Wang \\ Key Laboratory of Opto Elecronic Science and Technology \\ for Medicine of Ministry of Education \\ College of Photonic and Electronic Engineering Fujian \\ Normal University \\ Fujian, China \\ L. Hou \\ Key Laboratory of Opto Elecronic Science and Technology \\ for Medicine of Ministry of Education \\ College of Photonic and Electronic Engineering Fujian \\ Normal University \\ Fujian, China
}

\begin{abstract}
Fruit images segmentation is one of the important issues for fruit recognition. Inspired by the retina structure, a colour perception neural network is proposed to extract multiple colour features, and is applied to tomato fruits segmentation under natural complex background. The network is composed three layers. Input layer is colour perception layer inspired by the retina. The multiple colour space features based on retina structure and gray information are extracted. The middle layer is a back propagation neural network. The output layer is the segmented image. The experimental results show that the network can be used to well segment mature or immature tomato fruits from colour images with complex background, and eliminate the holes of segmentation image due to fruit surface refection. The proposed network is promising to be applied to fruit recognition of robots.
\end{abstract}

Keywords-fruit images segmentation; retina structure; multiple colour space; neural network

\section{INTRODUCTION}

Fruit picking robot is regarded as a potential replacement to release human beings from fruit harvest operation. The key of fruit picking robot is able to segment fruit from the complex background rapidly and accurately. Images segmentation in computer vision and image processing technology is one of the most difficult tasks. In recent years, there are many published research about the fruit images segmentation methods. For example, thresholding for Image segmentation, watershed transformation, K-means clustering algorithm, neural networks, and so on. Shah-Hosseini [1] used multilevel thresholding method to segment gray-level images. Dadwal et al.[2], operated directly on RGB(red-green-blue ) colour space without colour space transformation for apple image segmentation. The basic idea was based on thresholding method. Guo et al.[3], developed a threshold based algorithm with the R-B feature to segment red strawberry for harvesting robot. Ohta colour space was selected in this method. Annamalai et al.[4] reported a machine vision system to identify citrus fruits and to estimate yield information of citrus groves. The threshold of segmentation of the images to recognise citrus fruits was estimated from the pixel distribution in the HIS (hue-saturation-intensity) colour plane. Watershed transformation is one of the oldest segmentation techniques which were initially due to Beucher and Lantuejoul. Belaid et al.[5] proposed a new method based on a fast topological gradient algorithm and a watershed transformation to deal with the gray level image over-segmentation problem. Mente [6] used K-means clustering algorithm on the L*a*b colour space to segment colour fruit image. Jaffar et al.[7] also converted RGB to $\mathrm{L}^{*} \mathrm{a} * \mathrm{~b}$ colour space and used K-means clustering to segment Fresh Fruit Bunches images. Zhang et al.[8] used BP (Back Propagation) neural network to segment colour images. Because of R, G and B components strongly influencing by light, cucumber fruit image was transformed into HIS space.

Even though all these methods segment the fruit images effectively, the result of segmentation images based on single colour space always have many holes and incomplete due to fruit surface reflection. Biological evidence shows that the retina contains two forms of photosensitive neurons, i.e. rods and cones [9]. Rod cells are highly sensitive to light and respond in dim light and dark conditions. Three types of cones have primary sensitivity of red, green, and blue lights. Inspired 
by the roles of rods and cones in retina, the proposed approach in this paper has used multiple colour space combination based on retina structure and colour perception neural network. Multiple colour space combination not only reduces the illumination effect and enhances image colour information, but also removes holes in the segmented image. Therefore, accuracy of segmentation of fruit images can be improved.

This paper is organized as follows. In section 2, a method of fruit images segmentation and the architecture of the neural network are proposed. In section 3, algorithm for proposed network is introduced. In section 4 , some experimental results are presented. Finally, some conclusions and a topic for further study are given in section 5 .

\section{ARchitecture of THE Proposed Network}

The human visual system is quite complicated. It extracts various features through different parts. In the visual pathway, photoreceptors in the retina are the first level of neurons, including cone and rod cells. There are various receptive field of photoreceptor cells directly or indirectly affect certain nerve cell [10]. Inspired by retina structure and receptive field, a colour perception neural network is proposed. The architecture of neural network is shown in fig.1. In this model, five features are extracted as the input of the neural network for colour pixel $\mathrm{P}(\mathrm{x}, \mathrm{y}) . \mathrm{r}\left(\mathrm{x}^{\prime}, \mathrm{y}^{\prime}\right), \mathrm{g}\left(\mathrm{x}^{\prime}, \mathrm{y}^{\prime}\right), \mathrm{b}\left(\mathrm{x}^{\prime}, \mathrm{y}^{\prime}\right)$ are components in RGB colour space , $i\left(x^{\prime}, y^{\prime}\right)$ is component in HIS colour space, and $n\left(x^{\prime}, y^{\prime}\right)=0.2989 r\left(x^{\prime}, y^{\prime}\right)+0.587 g\left(x^{\prime}, y^{\prime}\right)+0.114 b\left(x^{\prime}, y^{\prime}\right)$ is gray level. In input layer, $\mathrm{R}(\mathrm{x}, \mathrm{y}), \mathrm{G}(\mathrm{x}, \mathrm{y}), \mathrm{B}(\mathrm{x}, \mathrm{y}), \mathrm{I}(\mathrm{x}, \mathrm{y})$ are neurons based on cones corresponding to receive field $\mathrm{RCx}, \mathrm{y}$, $\mathrm{N}(\mathrm{x}, \mathrm{y})$ is neuron based on rods corresponding to receive field RNx,y, $A$ and $B$ are areas of receive field $(A<B)$, the expressions as follow eqns.(1)-(5):

$$
\begin{aligned}
& R_{x, y}=\frac{1}{A} \sum_{\left(x^{\prime}, y\right) \in R C_{x, y}} r\left(x^{\prime}, y^{\prime}\right) \\
& G_{x, y}=\frac{1}{A} \sum_{\left(x^{\prime}, y\right) \in R C_{x, y}} g\left(x^{\prime}, y^{\prime}\right) \\
& B_{x, y}=\frac{1}{A} \sum_{\left(x^{\prime}, y\right) \in R C_{x, y}} b\left(x^{\prime}, y^{\prime}\right) \\
& I_{x, y}=\frac{1}{A} \sum_{\left(x^{\prime}, y\right) \in R C_{x, y}}\left(r\left(x^{\prime}, y^{\prime}\right)+g\left(x^{\prime}, y^{\prime}\right)+b\left(x^{\prime}, y^{\prime}\right)\right) / 3 \\
& N_{x, y}=\frac{1}{B} \sum_{\left(x^{\prime}, y^{\prime}\right) \in R N_{x, y}} n\left(x^{\prime}, y^{\prime}\right)
\end{aligned}
$$

Five neurons in input layer are corrected to hidden layer, and hidden layer has five neurons correcting to neuron $\mathrm{O}(\mathrm{x}, \mathrm{y})$ in the output layer of neural network . $\mathrm{O}(\mathrm{x}, \mathrm{y})$ has dimension as same as input image and can be assigned as classification numbers. The neural network can be trained to segment colour fruit images, as equation.(6):

$$
O(x, y)=\operatorname{NET}\left(R_{x, y}, G_{x, y}, B_{x, y}, I_{x, y}, N_{x, y}\right)
$$

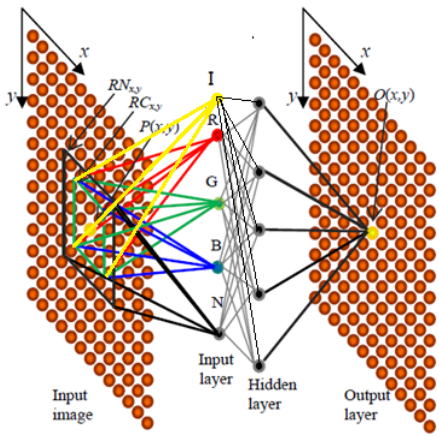

FIGURE I. ARCHITECTURE OF COLOUR PERCEPTION NEURAL NETWORK.

\section{Algorithm FOR THE PROPOSED NetWorK}

The training dataset contains two categories pixels, one is sampled from fruit areas of colour image, and the other is sampled from background areas of colour image. The received field of RCx,y is selected as $3 \times 3$ pixels and $R N x, y$ is selected as $5 \times 5$ pixels. These receptive fields can be adjusted according to the different images. But the larger the receptive fields, the more immune to noise, with trade off, loss of the sharpness at the border of the object [10]. Detailed procedures of the method are described as follows:

Step1: Sample training dataset.

1.Load colour fruit image, select one pixel $\mathrm{P}(\mathrm{x}, \mathrm{y})$ from fruit areas or background as training data, and at the same time label this pixel with classification numbers, background pixel is label as ' 0 ', fruit pixel is label as ' 1 '.

2.Five features are computed for each pixel.

$$
\begin{gathered}
\text { For } \mathrm{j}=\mathrm{y}-1: \mathrm{y}+1 \\
\begin{array}{c}
\text { For } \mathrm{i}=\mathrm{x}-1: \mathrm{x}+1 \\
\mathrm{p} 1=\mathrm{p} 1+\mathrm{P}(\mathrm{i}, \mathrm{j}, 1) ; \\
\mathrm{p} 2=\mathrm{p} 2+\mathrm{P}(\mathrm{i}, \mathrm{j}, 2) ; \\
\mathrm{p} 3=\mathrm{p} 3+\mathrm{P}(\mathrm{i}, \mathrm{j}, 3) ; \\
\mathrm{p} 4=\mathrm{p} 4+(\mathrm{p} 1+\mathrm{p} 2+\mathrm{p} 3) / 3
\end{array}
\end{gathered}
$$

end

end

For $\mathrm{j}=\mathrm{y}-2: \mathrm{y}+2$

$$
\begin{aligned}
& \text { For } \mathrm{i}=\mathrm{x}-2: \mathrm{x}+2 \\
& \mathrm{p} 5=\mathrm{p} 5+\mathrm{n}(\mathrm{i}, \mathrm{j}) ;
\end{aligned}
$$

end

end

$\mathrm{p} 1=\mathrm{p} 1 / 9 ;$

$\mathrm{p} 2=\mathrm{p} 2 / 9$;

$\mathrm{p} 3=\mathrm{p} 3 / 9$;

$\mathrm{p} 4=\mathrm{p} 4 / 9$; 


$$
\text { p5= p4/25; }
$$

3.Calculate the output value $\mathrm{O}(\mathrm{x}, \mathrm{y})$.

Step2: Training of neural network with the training dataset.

1.Initial the 3 layers neural network.

net=newff(minmax(p),[5,1], \{'tansig','purelin' $\}$,'trainlm');

net.trainParam.show $=1000$;

net.trainParam.epochs $=5000$;

net.trainParam.goal $=0.03$;

net.trainParam.epochs $=5000$;

2.Training network with features input $\mathrm{p}$ and output $\mathrm{t}$, net is trained network, that be used to distinguish between areas of the test sample.

net=train(Net,p,t);

Step3: Finally, mask0 and mask1are mask map corresponding to background and fruit area. The area of objects are less than a minimum size threshold, that will be removed from the mask 0 and mask 1 respectively in order to remove the noise of fruit images segmentation. Using the following method, two areas can be separated.

1.Load original image $\mathrm{P}(\mathrm{x}, \mathrm{y})$ as testing dataset, using trained neural network to get output value $\mathrm{O}(\mathrm{x}, \mathrm{y})$.

2.Calculate mask and segment the image.

For $\mathrm{y}=1$ : $\operatorname{size}(\mathrm{P}, 2)$

For $\mathrm{x}=1: \operatorname{size}(\mathrm{P}, 1)$

if $\mathrm{O}(\mathrm{x}, \mathrm{y})=0 \quad \operatorname{mask} 0(\mathrm{x}, \mathrm{y})=1$; else $\operatorname{mask} 1(\mathrm{x}, \mathrm{y})=1$; end

background $(\mathrm{x}, \mathrm{y})=\operatorname{mask} 0(\mathrm{x}, \mathrm{y}) * \mathrm{P}(\mathrm{x}, \mathrm{y})$;

fruit $(\mathrm{x}, \mathrm{y})=\operatorname{mask} 1(\mathrm{x}, \mathrm{y}) * \mathrm{P}(\mathrm{x}, \mathrm{y})$;

end

end

\section{RESULTS OF THE EXPERIMENTS}

The neural network is simulated in Matlab. As shown in fig.2, Training dataset is sampled from the original image about 40 points each area, squares are points of fruit, stars are points of background. Fig. 3 shows the network converges very fast during training process.

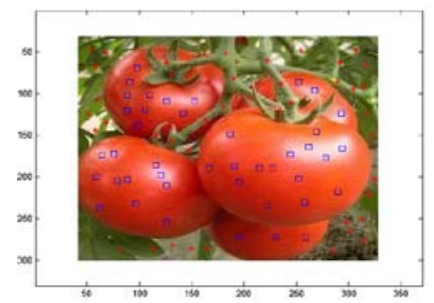

FIGURE II. SAMPLE TRAINING SET

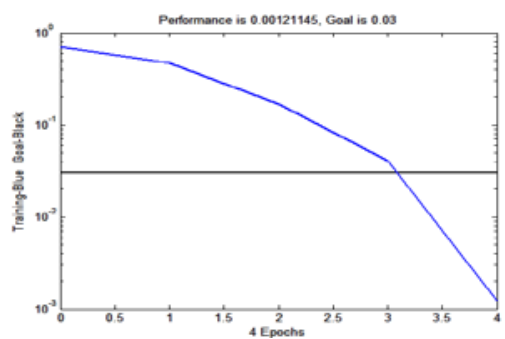

FIGURE III.

ERROR CHANGE IN TRAINING PROCESS

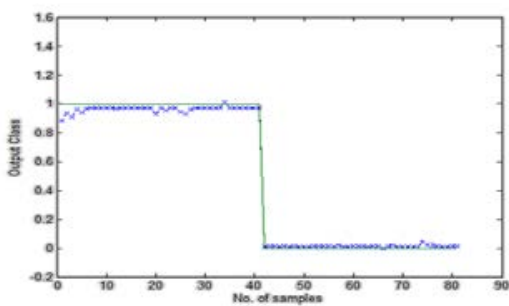

FIGURE IV. $\quad$ OUTPUTS OF NEURAL NETWORK

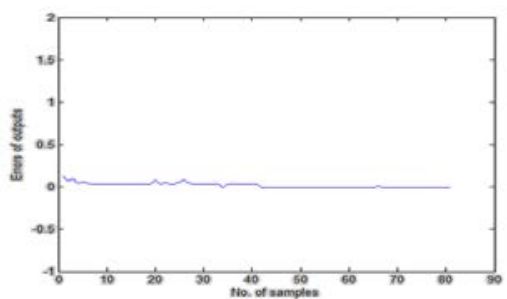

FIGURE V. ERRORS

Fig.4 depicts the output values corresponding to samples in the training dataset. Samples from No.1 to No.40 are fruit area, the output values are almost at ' 1 ', No.41 to No.80 are background, the values are almost at ' 0 ', and fig. 5 shows the errors of output are very small.

The network is applied to separate mature tomato fruits on the tree under natural illumination condition, as fig. 6 shown. Fruits are successfully detected. In Original tomatoes images, fruit surface have several bright spots due to reflection, but there are no holes in segmentation images. Fig.7 illustrates the results of different type tomatoes segmentation. Fig. 8 shows the results of immature tomatoes segmentation. The colour of immature tomatoes is similarly to the background. Fig.9 shows the result of segmentation image using the K-means clustering method, which has many holes at fruit areas.
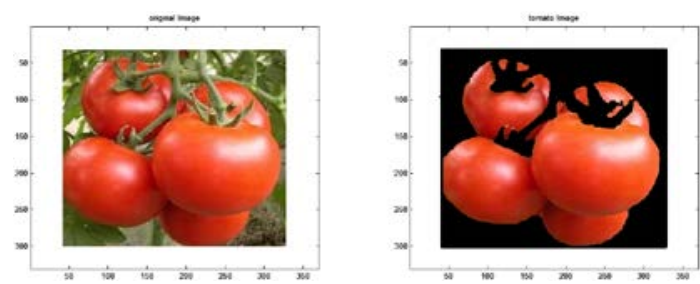

FIGURE VI.

ORIGINAL IMAGE AND SEGMENT IMAGE FOR MATURE TOMATOES 

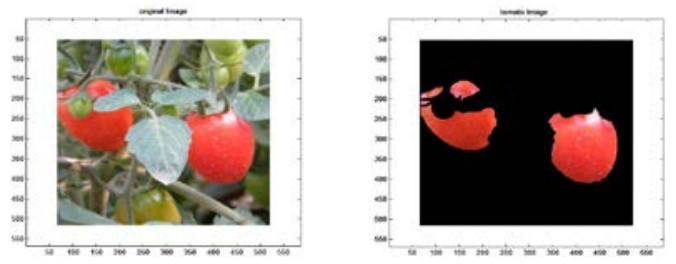

FIGURE VII.

ORIGINAL IMAGE AND SEGMENT IMAGE FOR OTHER TOMATOES
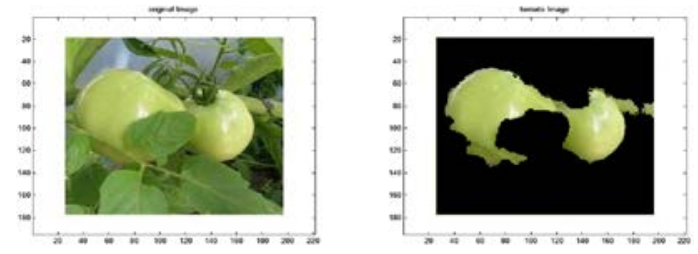

FIGURE VIII.

ORIGINAL IMAGE AND SEGMENT IMAGE FOR IMMATURE TOMATOES

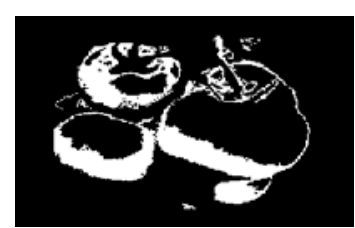

FIGURE IX.

$$
\begin{aligned}
& \text { K-MEANS CLUSTERING FOR SEGMENTATION } \\
& \text { IMAGE. }
\end{aligned}
$$

\section{CONCLUSION}

This paper presents a method for colour fruit images segmentation. Biological processing mechanism is used in the network. The advantage is multiple colour space features and gray information integrated to seperate fruits in a colour image. Therefore, the proposed approach is cable to identify fruits in the colour images and remove the holes owing to reflection. The experimental results show that the network is able to segment fruits on trees efficiently in despite of the complex background and vary illumination. Further study will consider the fruits classification, fruits yield estimation and fruits disease identification using proposed method.

\section{ACKNOWLEDGMENTS}

The authors gratefully acknowledge the fund from the Natural Science Foundation of China (Grant No.61179011 \& No. 61070062) and Science and Technology Major Projects for Industry-academic Cooperation of Universities in Fujian Province (Grant No.2013H6008).

\section{REFERENCES}

[1] Hamed Shah-Hosseini, Multilevel Thresholding for Image Segmentation using the Galaxy-based Search Algorithm. International Journal of Intelligent Systems and Applications, 11, pp.19-33, 2013.

[2] Meenu Dadwal \& Vijay Kumar Banga, Estimate Ripeness Level of fruits Using RGB color space and Fuzzy logic Technique. International Journal of Engineering and Advanced Technology, 2(1), pp.225-229, 2012.

[3] Feng Guo, Qixin Cao \& Masateru Nagata, Fruit Detachment and Classification Method for strawberry harvesting robot: the conversion from RGB to OHTA color space then fruit segmentation was achieved by threshold algorithm. International Journal of Advanced Robotic Systems, 5(1), pp. 41-48, 2008.
[4] Palaniappan Annamala,Won Suk Lee \& Thomas F. Burks, Color Vision System for Estimating Citrus Yield in Real-time. ASAE Annual International Meeting, pp.1-12.2004.

[5] Lamia Jaafar Belaid \& Walid Mourou, Image segmentation: a watershed transformation. Image Anal Stereol, 28,pp.93-102,2009.

[6] Rajivkumar Mente, Color image segmentation and recognition based on shape and color features.Computer Science Engineering, 3(1),pp.51$56,2014$.

[7] Ahmed Jaffar, Roseleena Jaafar \& Nursuriati Jamil, Photogrammetric Grading of Oil Palm Fresh Fruit Bunches. Mechanical \& Mechatronics Engineering,9(10), pp.7-13,2009.

[8] Libin Zhang, Qinghua Yang \& Yi Xun, Recognition of greenhouse cucumber fruit using computer vision. New Zealand journal of Agricultural Research, 50 (5), pp. 1293-1298,2007.

[9] Qingxiang Wu, Xi Huang \& Jianyong Cai, Segmentation of Leukocytes in Blood Smeare Images Using Color Processing Mechanism Inspired by The Visual System, Proc. Of the 2nd Int.Conf. On Biomedical Engineering and Informatics, BMEI,pp.368-372,2009.

[10] Qingxiang Wu,T.M.McGinnity \& G.D.Valderrama-Gonzalez, Colour Image Segmentation Based on a Spiking Neural Network Model Inspired by the Visual System, Springer-Verlag: Berlin Heidelberg,pp.49-57,2010. 